\title{
Estudo sobre as Condições Pedagógicas das Escolas Médicas Portuguesas: Uma Análise Nacional Sobre a Satisfação Estudantil, Rácios Estudante-Tutor e Número de Admissões
}

\section{Study on Portuguese Medical Schools' Learning Conditions: A National Analysis on Student Satisfaction, Student-Tutor Ratios and Number of Admissions}

\author{
Pedro Grilo DIOGO $\rrbracket^{1}$, Afonso MOREIRA ${ }^{1}$, Ana COIMBRA ${ }^{1}$, Ana SILVA ${ }^{1}$, Artur MARTINS ${ }^{1}$, Carlos MENDONÇA ${ }^{1}$, \\ Constança CARVALHO ${ }^{1}$, Gonçalo ALMEIDA ${ }^{1}$, Hugo ALMEIDA ${ }^{1}$, Inês MOREIRA ${ }^{1}$, Marta RODRIGUES ${ }^{1}$, Miguel GOULÃO ${ }^{1}$, \\ Rafael VASCONCELOS ${ }^{1}$, Rodrigo VICENTE ${ }^{1}$, Sara MAGANO ${ }^{1}$ \\ Acta Med Port 2016 May;29(5):301-309 - http://dx.doi.org/10.20344/amp.6795
}

\section{RESUMO}

Introdução: Os ambientes de ensino clínico e não clínico, bem como as condições de avaliação e estudo, influenciam a satisfação estudantil com as Escolas Médicas. Os rácios estudante-tutor podem ter impacto na perceção sobre o ensino em meio clínico. Este estudo tem como objetivo analisar a satisfação dos estudantes de Medicina e os rácios estudante-tutor em relação com o número de admissões das Escolas Médicas.

Material e Métodos: Foi criado um questionário sobre os ambientes de aprendizagem, avaliação e estudo em oito Escolas Médicas, distribuído a 2037 estudantes. Calculou-se o alfa de Cronbach (consistência interna) e executou-se uma análise de componentes principais.

Resultados: Condições de avaliação obtiveram os melhores resultados de satisfação, enquanto o ensino em meio clínico revelou as menores pontuações. O rácio estudante-tutor nacional em disciplinas clínicas $(7,53)$ traduz diferenças significativas entre Escolas. Instituições com maior número de admissões evidenciam resultados inferiores de satisfação estudantil $(r=-0,756 ; p<0,05)$, com redução progressiva ao longo do curso. Elevados rácios estão correlacionados com baixa satisfação com o ensino em meio clínico $(r=-0,826 ; p<0,05)$.

Discussão: O ensino em meio clínico evidencia menor satisfação estudantil, traduzindo os elevados rácios em disciplinas clínicas Dependendo do número de admissões, existem diferenças significativas entre Escolas. A qualidade das estratégias de ensino-aprendizagem e articulação hospitalar podem igualmente ser variáveis importantes.

Conclusão: As Escolas com maior número de admissões podem ser mais suscetíveis a baixos resultados de satisfação estudantil. Elevados rácios estudante-tutor em disciplinas clínicas podem reduzir a qualidade do ensino em meio clínico e inibir a aquisição de competências.

Palavras-chave: Alunos de Medicina; Educação Médica; Escolas Médicas; Portugal; Programas de Autoavaliação.

\section{ABSTRACT}

Introduction: Experiences of clinical and nonclinical learning environments, as well as assessment and study environments influence student satisfaction with their medical schools. Student-tutor ratios may impact on their perception of clinical learning environments. The aim of this study was to analyze medical students' satisfaction and student-tutor ratios in relation to medical schools' number of admissions.

Material and Methods: A questionnaire was created, regarding learning, assessment and study environments in eight medical schools. 2037 students participated in this cross-sectional study. Cronbach's alpha (internal consistency) was calculated and principal component analysis was conducted. Pearson correlations and multiple comparisons were analyzed.

Results: Assessment environments showed the highest satisfaction scores and clinical learning environments the lowest scores. The national student-tutor ratio in clinical rotations is 7.53; there are significant differences among schools. Institutions with higher number of admissions showed the lowest scores of overall student satisfaction $(r=-0.756 ; p<0.05)$, which decreased with progression in the medical course. High student-tutor ratios are strongly correlated with low levels of satisfaction regarding clinical learning environments $(r=-0.826 ; p<0.05)$

Discussion: Clinical learning environments show the lowest satisfaction scores, which may expose the effect of high ratios in clinical rotations. Depending on the number of admissions, significant differences between medical schools were found. Quality of teachinglearning strategies and articulation with hospitals might also be important variables.

Conclusion: Medical schools with more admissions might be more susceptible to lower scores of student satisfaction. High studenttutor ratios in clinical rotations may reduce the quality of learning experiences and inhibit the acquisition of competences.

Keywords: Medical Education; Medical Schools; Medical Students; Portugal; Self-Evaluation Programs.

\footnotetext{
1. Grupo de Trabalho em Educação Médica. Associação Nacional de Estudantes de Medicina. Portugal.

$\triangle$ Autor correspondente: Pedro Grilo Diogo. pgrilodiogo@gmail.com

Recebido: 03 de agosto de 2015 - Aceite: 28 de dezembro de 2015 | Copyright @ Ordem dos Médicos 2016
} 


\section{INTRODUÇÃO}

A gestão racional dos recursos humanos em Saúde é uma necessidade considerada premente pelos organismos internacionais, visando a formação de profissionais em número e qualidade ajustadas ao propósito de alcançar serviços de Saúde que sirvam as necessidades da população. ${ }^{1,2}$ Esta gestão requer a avaliação das necessidades previsionais de médicos a nível regional/nacional, ${ }^{3}$ bem como uma avaliação da capacidade formativa das Escolas Médicas, que depende da adequação das suas condições pedagógicas, condicionada pelo número de estudantes admitidos anualmente e que frequentam a Escola, e pelos seus recursos financeiros, humanos e materiais (corpo docente, Hospitais de ensino, infraestruturas, materiais pedagógicos). ${ }^{4}$

Os questionários formais aos estudantes são amplamente utilizados para avaliar as condições pedagógicas no Ensino Superior. O feedback estudantil permite obter importantes informações para avaliar condições pedagógicas e desenvolver iniciativas para melhorar a qualidade do ensino. ${ }^{5}$

No ensino superior, os rácios estudante-tutor (RET) são interpretados enquanto indicadores da qualidade do ensino: baixos RET sugerem uma apreciação positiva das condições pedagógicas das instituições de ensino superior ainda que representem apenas parcialmente a realidade educativa, variando com as metodologias de ensino-aprendizagem e ambientes de aprendizagem. ${ }^{6}$ De facto, as Escolas Médicas devem estabelecer um número de ingressos que respeite a sua capacidade formativa máxima e atentar aos RET enquanto indicadores de qualidade. ${ }^{4}$

O número de estudantes admitidos nas Escolas Médicas portuguesas tem sofrido um incremento considerável: desde 1990, em termos relativos, Portugal foi o país da Organisation for Economic Cooperation and Development (OCDE) que mais aumentou o número de diplomados em Medicina. ${ }^{7}$ Tendo em consideração o reconhecimento internacional sobre a relevância de planear de forma integrada e sustentável a formação de médicos, importa estudar a realidade portuguesa, analisando se as Escolas Médicas estão dotadas de capacidade formativa adequada ao atual número de ingressos por ano.

O presente trabalho tem como objetivo analisar dois indicadores da capacidade formativa das Escolas Médicas portuguesas, nomeadamente a satisfação estudantil em várias componentes do ambiente educativo e os RET no ensino em meio clínico, analisando a correlação destes indicadores com o número de ingressos de cada Escola. Este estudo divide-se em dois componentes: 1) apreciação das condições pedagógicas das Escolas Médicas, através de questionários de satisfação sobre as condições pedagógicas das Escolas e a 2) apreciação das condições pedagógicas para o ensino clínico, calculando os RET das Escolas Médicas.

\section{MATERIAL E MÉTODOS}

\section{Questionários sobre as condições pedagógicas das} Escolas Médicas portuguesas

\section{a) Descrição dos questionários}

Foram construídos questionários sobre as condições pedagógicas das Escolas Médicas constituídos por seis grupos de questões, nomeadamente: Condições de Avaliação, Locais de Estudo, Aulas Teóricas/Seminários, Aulas teórico-práticas/Casos Clínicos/Resolução de problemas/ Tutorias, Aulas práticas/laboratoriais e Ensino em Meio Clínico. Dentro de cada um destes grupos de questões, existem entre 4 a 6 alíneas cuja resposta foi dada numa escala de Likert pontuada de 1 a 6 (1: completamente insatisfeito; 6: completamente satisfeito), existindo ainda a opção "Sem opinião".

Nos anos letivos de 2011/2012 (ano de 2012) e de 2013/2014 (ano de 2013) foram aplicados questionários a estudantes de Medicina de todos os anos curriculares de cada uma das Escolas Médicas portuguesas, nas instalações das respetivas Escolas aquando de aulas teóricas. Em 2012, os questionários foram aplicados em formato de papel entre janeiro e fevereiro. Em 2013, os questionários foram aplicados em formato de papel e em formato eletrónico (disponibilizado através de grupos de Facebook

Tabela 1 - Número de questionários aplicados e respetiva percentagem em relação ao número total de estudantes, por ano letivo e por Escola Médica

\begin{tabular}{|c|c|c|c|c|c|c|}
\hline & Escola & $2011 / 2012$ & $\%(2011)$ & 2012 / 2013 & $\%(2013)$ & Tota \\
\hline 1 & ECS-UM & 148 & 26,6 & 203 & 25,1 & 351 \\
\hline 2 & FMUP & 339 & 20,4 & 277 & 17,8 & 616 \\
\hline 3 & ICBAS & 214 & 19,7 & 294 & 27,1 & 508 \\
\hline 4 & FCS-UBI & 149 & 19,8 & 192 & 19,9 & 341 \\
\hline 5 & FMUC & 342 & 20,1 & 341 & 19,6 & 683 \\
\hline 6 & FMUL & 386 & 17,9 & 412 & 19,0 & 798 \\
\hline 7 & NMS|FCM & 324 & 20,6 & 292 & 17,4 & 616 \\
\hline \multirow[t]{2}{*}{8} & DCBM-UAlg & 19 & 20,7 & 30 & 21,4 & 49 \\
\hline & Total & 1921 & & 2041 & & 3962 \\
\hline
\end{tabular}


e mailing lists) durante o mês de outubro e início do mês de novembro.

O questionário foi aplicado a uma amostra aleatória de cerca de $20 \%$ do número total de estudantes de cada ano curricular, em cada uma das Escolas Médicas (Tabela 1). Todos os questionários implementados foram preenchidos pelos estudantes e contabilizados para efeitos de análise estatística. Comparando ambos os grupos de questionários, referentes a 2012 e a 2013, o teste de qui-quadrado mostra uma distribuição idêntica do número de questionários por ano curricular e por Escola Médica $(p>0,05)$.

\section{b) Tratamento e análise dos dados}

Os questionários foram analisados por leitura ótica no Centro de Educação Médica da Faculdade de Medicina da Universidade do Porto (FMUP) em equipamento Fujitsu fi-5120c ${ }^{\circledR}$. Os seus resultados foram transferidos para bases de dados nos softwares IBM SPSS Statistics $19^{\circledR} \mathrm{e}$ Microsoft Office Excel $2010^{\circledR}$. A fiabilidade do questionário foi avaliada através do cálculo da sua consistência interna pelo alpha de Cronbach. Os testes de Kaiser-Meyer-Olkin e o teste de esfericidade de Bartlett analisaram a adequação das questões para a aplicação da análise de componentes principais; esta análise foi conduzida com rotação de varimax para identificar o número de componentes sugeridos pelos dados e para identificar quais as perguntas que pertenciam a cada componente. Considerou-se que a associação entre as perguntas e os componentes principais era forte quando a correlação era superior a 0,70 , moderada entre 0,30 e 0,70 e fraca se inferior a 0,30. De acordo com os dados obtidos, todas as questões tiveram uma relação no mínimo satisfatória, pelo que todas incluíram a análise de componentes principais. As questões do questionário foram agregadas em quatro componentes: C1 - ensino em meio não clínico, C2 - ensino em meio clínico, C3 - condições de avaliação e C4 - condições de ensino. Para o cálculo da pontuação de cada Escola Médica em cada um dos componentes foi utilizada a média das pontuações dos estudantes nas perguntas agregadas. Para o cálculo da média das pontuações no componente $\mathrm{C} 2$, apenas foram consideradas as respostas de estudantes do terceiro ano curricular ou superior.

\section{Rácios estudante-tutor das Escolas Médicas portugue- sas}

Os RET traduzem o quociente entre o número de estudantes e o número de tutores numa determinada Unidade Curricular (UC). Para efeitos de simplificação, no presente Estudo, os rácios estudante-tutor serão representados pelos resultados dos quocientes estudantes/tutor (por exemplo, um rácio de cinco estudantes para um tutor equivale a um rácio de cinco). Os RET foram calculados em todas as Escolas Médicas em Portugal (com exceção do Departamento de Ciências Biomédicas e Medicina da Universidade do Algarve (DCBM-UAlg) em que não foi possível recolher dados), para todas as UCs com componente clínica (do terceiro ano curricular e superior) e simultaneamente com sistema tutorial, de abril a dezembro de 2014. Considerou-se que uma UC pratica um sistema tutorial quando a cada grupo de estudantes, faz corresponder um tutor responsável pelo ensino em meio clínico, durante a maioria do tempo letivo, com responsabilidade na avaliação dos estudantes a seu cargo. Para o cálculo dos RET foram utilizadas metodologias de recolha de dados adequadas a cada Escola Médica, nomeadamente: 1) Pedido de dados aos Serviços Académicos/Divisões de Educação Médica das Escolas; 2) Análise de documentos oficiais, distribuídos aos estudantes de cada UC pelas suas Regências; 3) Solicitação de dados sobre os RET aos estudantes das Associações/Núcleos locais, Comissões de Curso ou estudantes de cada turma/ bloco/rotação. Os RET obtidos foram enviados aos ConseIhos Pedagógicos das várias Escolas Médicas, juntamente com um pedido para a sua homologação. A homologação foi conferida pelos Conselhos Pedagógicos da Escola de Ciências Médicas da Universidade do Minho (ECS-UM), Faculdade de Ciências da Saúde da Universidade da Beira Interior (FCS-UBI) e FMUP.

\section{RESULTADOS}

a. Questionários sobre as condições pedagógicas das Escolas Médicas Portuguesas

Análise global

Tabela 2 - Número de respostas obtidas, média por ano de implementação dos questionários e média global por componente (de C1 a C4) e respetivo construto

\begin{tabular}{|c|c|c|c|c|c|c|c|}
\hline \multirow[b]{2}{*}{ Componente } & \multirow[b]{2}{*}{ Construto } & \multicolumn{3}{|c|}{2012} & \multicolumn{3}{|c|}{2013} \\
\hline & & $\mathrm{n}$ & Média & IC $95 \%$ & $\mathrm{n}$ & Média & IC $95 \%$ \\
\hline $\mathrm{C} 1$ & $\begin{array}{l}\text { Ensino em meio não clínico } \\
\text { (teórico, teórico-prático e } \\
\text { prático-laboratorial) }\end{array}$ & 1914 & 3,990 & $3,951-4,028$ & 2070 & 3,969 & $3,932-4,007$ \\
\hline $\mathrm{C} 2$ & Ensino em meio clínico & 1148 & 3,688 & $3,631-3,745$ & 1323 & 3,676 & $3,622-3,730$ \\
\hline C3 & Condições de avaliação & 1911 & 4,300 & $4,262-4,338$ & 2061 & 4,252 & $4,215-4,289$ \\
\hline \multirow[t]{2}{*}{$\mathrm{C} 4$} & Condições de estudo & 1911 & 3,711 & $3,661-3,761$ & 2070 & 3,857 & $3,814-3,900$ \\
\hline & & & $(3,922)$ & & & $(3,938)$ & \\
\hline
\end{tabular}


Analisando os anos de 2012 e 2013 (Tabela 2), respetivamente, é possível verificar que as condições de avaliação $(4,300$ e 4,252) representam a componente com avaliação mais elevada, seguindo-se o ensino em meio não clínico $(3,990$ e 3,969$)$, as condições de estudo $(3,711$ e 3,857$)$ e por fim o ensino em meio clínico $(3,688$ e 3,676$)$, que representa o componente com pior avaliação média. Analisando todos os componentes, verifica-se uma avaliação global média das condições pedagógicas nas Escolas Médicas de 3,922 em 2012 e 3,938 em 2013.

\section{Ensino em meio não clínico}

No componente $\mathrm{C} 1$, podemos constatar a existência de três Escolas Médicas com resultados médios superiores a 4,50, nomeadamente o DCBM-UAlg, a FCS-UBI e a ECS-UM, com diferenças estatisticamente significativas em relação às restantes Escolas em ambos os anos $(p<$ 0,05). FMUP, Instituto de Ciências Biomédicas Abel Salazar (ICBAS), Faculdade de Medicina da Universidade de Lisboa (FMUL) e Nova Medical School | Faculdade de Ciências Médicas (NMS|FCM) apresentam resultados idênticos ligeiramente inferiores aos 4,00 pontos, enquanto que a Faculdade de Medicina da Universidade de Coimbra (FMUC) é a Escola com pior classificação em ambos os anos, pontuando 3,657 em 2012 e 3,281 em 2013. Em 2013, esta diferença é estatisticamente significativa em relação a todas as outras Escolas Médicas.

\section{Ensino em meio clínico}

No componente $\mathrm{C} 2\left(3^{\circ}, 4^{\circ}, 5^{\circ}\right.$ e $6^{\circ} \mathrm{s}$ anos curriculares), analisando ambos os anos de 2012 e 2013, o DCBM-UAlg apresenta os valores médios mais elevados, seguido da ECS-UM, FCS-UBI e da NMS|FCM (em 2013), com diferenças estatisticamente significativas em relação às restantes Escolas ( $p<0,05$, ver Fig. 1). A FMUP foi a Escola com pior classificação em 2012, pontuando 3,443. Já a FMUC foi a Escola com pior classificação em 2013, pontuando 3,194; esta diferença em 2013 é estatisticamente significativa em relação a todas as outras Escolas Médicas.

\section{Condições de avaliação}

No componente $\mathrm{C} 3$, analisando conjuntamente os anos de 2012 e 2013, podemos constatar a existência de três Escolas Médicas com resultados médios superiores a 4,50: o DCBM-UAlg, a FCS-UBI e a ECS-UM, com diferenças estatisticamente significativas para as restantes Escolas em ambos os anos (excetuando o DCBM-UAlg em 2013) $(p<0,05)$. As restantes Escolas, com valores médios idênticos entre os 3,90 e 4,20, não apresentam diferenças estatisticamente significativas entre si, em qualquer um dos anos estudados.

\section{Condições de estudo}

No componente $\mathrm{C} 4$, analisando conjuntamente os anos de 2012 e 2013, podemos constatar a existência de três Escolas Médicas com resultados médios superiores a 4,50, nomeadamente a ECS-UM, a FCS-UBI e o DCBM-UAlg (excetuando 2013), com diferenças estatisticamente significativas para as restantes Escolas em ambos os anos ( $p<$ $0,05)$. De seguida, em 2013, surge a FMUP $(4,097)$, com diferença significativa para as restantes Escolas, nomeadamente a FMUL, a NMS|FCM, o ICBAS e, por fim, a FMUC, tendo esta a pior classificação neste ano, pontuando 3,517. Em 2012, a NMS|FCM tinha a pior classificação média $(2,892)$.

\section{Satisfação global, por Escola Médica}

Analisando a Satisfação Global, no ano de 2013, podemos constatar a existência de três Escolas Médicas com resultados médios superiores a 4,50 , que constituem um grupo sem diferenças estatisticamente significativas entre si [DCBM-UAlg $(4,633)$, ECS-UM $(4,528)$ e FCS-UBI $(4,515)]$. Este grupo é diferente $(p<0,05)$ de outro grupo de Escolas (NMS|FCM, FMUP, ICBAS e FMUL), que por sua vez é significativamente diferente da FMUC, a Escola com pior classificação $(3,511)$ (Fig. 2).

\section{Evolução dos componentes ao longo dos anos cur- riculares}

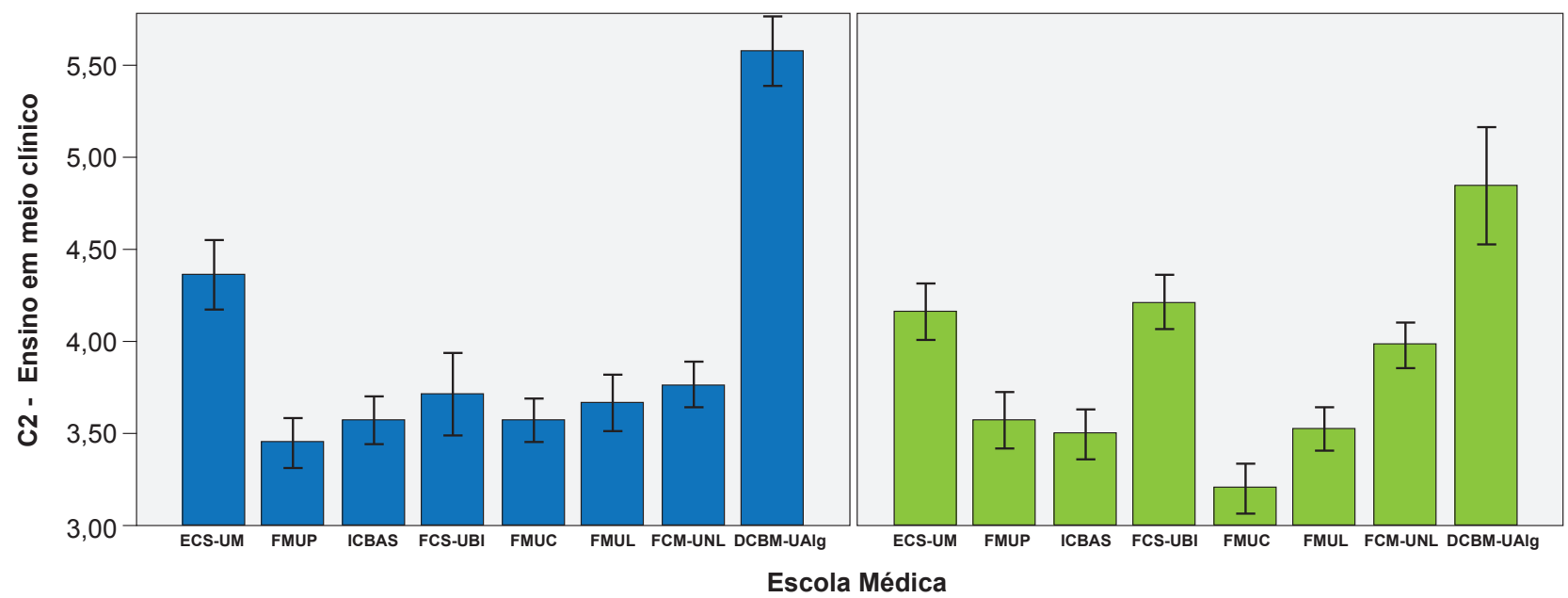

Figura 1 - Valores médios do componente C2, por ano de implementação dos questionários e por Escola Médica. A azul, o gráfico correspondente a 2012 e, a verde, o gráfico correspondente a 2013. Intervalos de confiança a 95\%. 


\begin{tabular}{rcccr}
\hline & Escola & Média & \multicolumn{2}{c}{ Desvio-padrão } \\
\hline 1 & ECS-UM & 4,528 & 0,871 & $2,3,5,6,7$ \\
2 & FMUP & 4,104 & 0,958 & $1,3,4,8$ \\
3 & ICBAS & 3,916 & 0,861 & $1,2,4,8$ \\
4 & FCS-UBI & 4,515 & 0,831 & $2,3,5,6,6,7$ \\
5 & FMUC & 3,511 & 0,868 & $1,2,3,4,6,6,7$ \\
6 & FMUL & 3,889 & 0,834 & $1,4,5,7,8$ \\
7 & NMS|FCM & 4,109 & 0,818 & $1,4,5,6,6$ \\
8 & DCBM-UAlg & 4,633 & 0,964 & $2,3,5,5,6,7$ \\
& Total & 3,873 & $p>0,001$ \\
\hline
\end{tabular}

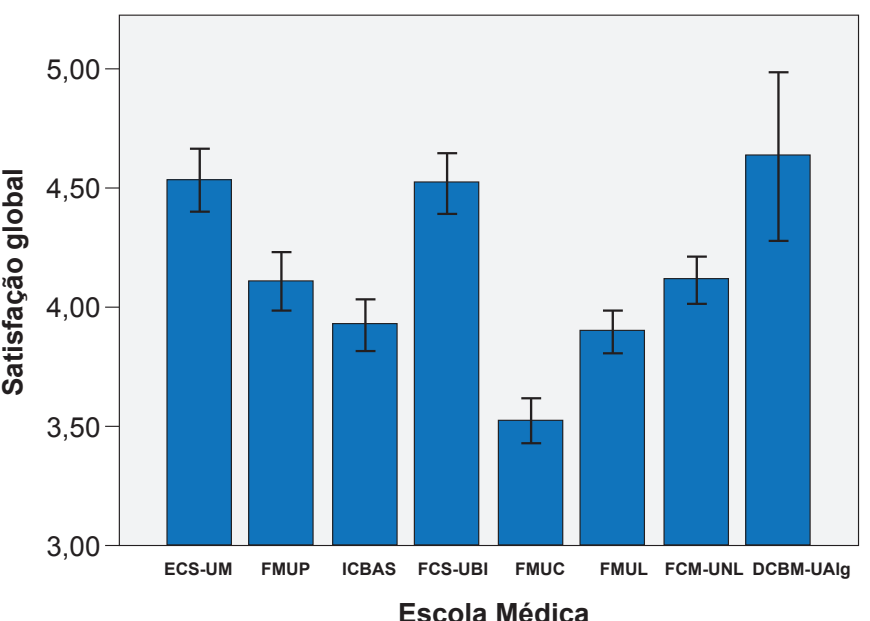

Figura 2 - Valores médios da Satisfação Global (com desvio-padrão), por Escola Médica, e valor médio global (ponderado pelo número de ingressos) de todas as Escolas. Gráfico com intervalos de confiança a 95\%. Para um $p<0,001$, o teste de ANOVA evidencia diferenças entre Escolas. As diferenças específicas entre Escolas são indicadas pelas letras em sobreescrito (teste de Bonferroni).

Analisando a evolução dos quatro componentes ao longo do curso de Medicina, tanto em 2012 como em 2013, podemos constatar que existem em ambos os anos: 1) diferenças significativas entre Escolas Médicas $(p<0,05), 2)$ diferenças significativas entre anos curriculares $(p<0,05$; exceto $(2)$, e 3 ) diferenças significativas entre Escolas no que respeita ao padrão de evolução dos componentes ao longo dos anos curriculares $(p<0,05)$. Com a progressão nos anos curriculares, do primeiro para o sexto ano, existe uma tendência geral decrescente nas médias dos componentes. No entanto, a ECS-UM, a FCS-UBI e o DCBM-UAlg mantêm uma pontuação semelhante ao longo dos anos curriculares nos componentes $\mathrm{C} 1, \mathrm{C} 3$ e $\mathrm{C} 4$, não evidenciando a tendência decrescente das restantes Escolas.

\section{Satisfação global por Escola Médica e em relação com o seu número de ingressos}

Uma análise estatística da correlação entre as pontuações médias de cada Escola Médica na Satisfação Global e o seu número de ingressos no ano de 2013 revela uma correlação negativa, estatisticamente significativa ( $p$ $<0,05)$, entre a Satisfação Global e o número de ingressos (coeficiente de Pearson de -0,756) (Fig. 3). Assim, as Escolas com maior número de ingressos têm tendencialmente uma menor Satisfação Global por parte dos estudantes com os cursos de Medicina. Dividindo o gráfico apresentado em quatro quadrantes, verificamos que: 1) 0 DCBM-UAlg, a ECS-UM e a FCS-UBI apresentam-se no quadrante superior esquerdo, correspondente às Escolas com menor número de ingressos e maior Satisfação Global dos estudantes; 2) O ICBAS surge na transição entre o quadrante superior esquerdo e o quadrante inferior esquerdo, revelando baixo número de ingressos e Satisfação Global dos estudantes sobre a média nacional; 3) A NMS|FCM e a FMUP surgem no quadrante superior direito, apresentando elevado número de ingressos mas Satisfação Global acima da média; 4) A FMUC e a FMUL (na transição de quadrantes) aparecem no quadrante inferior direito, com elevado número de ingressos e Satisfação Global abaixo da média.

\section{Rácios estudante-tutor das Escolas Médicas portu- guesas}

a) Rácios estudante-tutor por Escola Médica

O rácio estudante-tutor médio nos $3^{\circ}, 4^{\circ}$ e $5^{\circ} \mathrm{s}$ anos curriculares, ponderado pelo número de admissões de cada Escola, é de 7,53. Os valores para os sextos anos curriculares são mais reduzidos, entre 1,03 (FCS-UBI) e 2,00 (FMUP e FMUC) (Fig. 4). Após análise estatística dos RET médios às UCs dos $3^{\circ}, 4^{\circ}$ e $5^{\circ} \mathrm{s}$ anos curriculares, existem diferenças significativas entre Escolas Médicas $(p<0,05)$ e diferenças signficativas entre UCs $(p<0,05)$ dos $3^{\circ}, 4^{\circ}$ e $5^{\circ}$ s anos curriculares. Constata-se que $57,5 \%$ das diferenças dos RET são explicadas por diferenças efetivas entre Escolas. Por outro lado, $11,9 \%$ da variação dos rácios é explicada por diferenças específicas entre UCs. Por fim, $30,7 \%$ da variação é explicada por fatores inespecíficos que não as diferenças entre Escolas ou entre UCs.

\section{b. Relação entre as pontuações médias do compo- nente C2 e os rácios estudante-tutor, por Escola Médi-} ca

Uma análise estatística da correlação entre os RET médios de cada Escola Médica nos $3^{\circ}, 4^{\circ}$ e $5^{\circ} \mathrm{s}$ anos curriculares e as suas pontuações médias no componente $\mathrm{C} 2$ do ensino em meio clínico revela uma correlação negativa, estatisticamente significativa $(p<0,05)$, entre a pontuação média no componente $\mathrm{C} 2$ e o RET médio (coeficiente de Pearson de -0,826) (Fig. 5). Assim, as Escolas Médicas com maiores RET apresentam os menores valores de satisfação estudantil com o ensino em meio clínico. Dividindo o gráfico apresentado em quatro quadrantes, verifica-se que: 1) A ECS-UM, a FCS-UBI e a NMS|FCM apresentam-se no quadrante superior esquerdo, correspondente às Escolas com menor rácio estudante-tutor e maior satisfação estudantil; 2) A FMUP e a FMUL (na transição) surgem no quadrante inferior esquerdo, revelando RET abaixo da mé- 


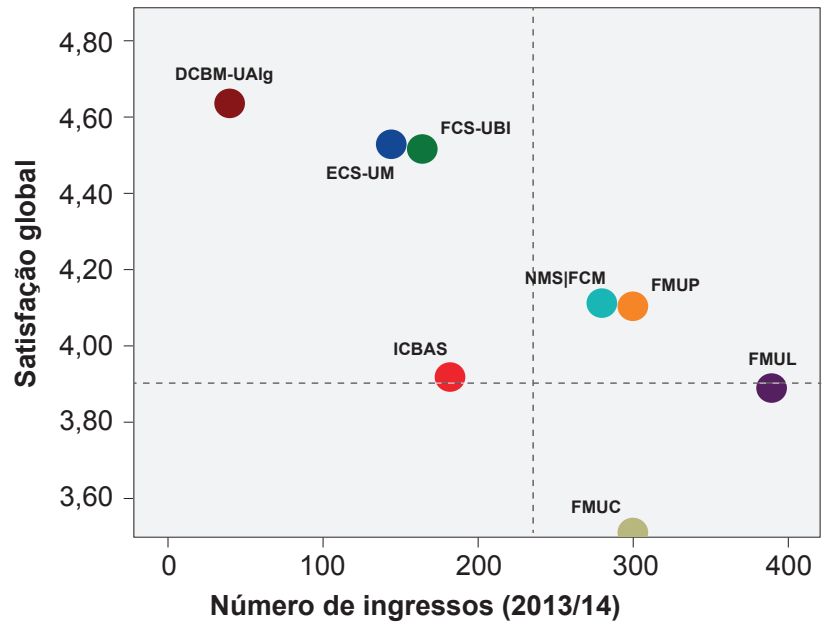

Figura 3 - Interação entre a pontuação média de Satisfação Global e o número de ingressos, no ano letivo 2013/2014, por Escola Médica. A reta tracejada na vertical cruza o eixo das abcissas no valor médio de ingressos das Escolas Médicas em Portugal (226) e a reta tracejada na horizontal cruza o eixo das ordenadas no valor médio da Satisfação Global (ponderado pelo número de ingressos) das Escolas Médicas $(3,873)$.

dia mas satisfação estudantil baixa; 3) O ICBAS e a FMUC surgem no quadrante inferior direito, com elevados RET e satisfação estudantil abaixo da média.

\section{c. Relação entre o número de ingressos e os rácios estudante-tutor, por Escola Médica}

Uma análise estatística da correlação entre os RET de cada Escola Médica nos $3^{\circ}, 4^{\circ}$ e $5^{\circ} \mathrm{s}$ anos curriculares e o seu número de ingressos no ano letivo de 2013/2014 não evidencia uma correlação estatisticamente significativa $(p>0,05)$. Dividindo o gráfico apresentado em quatro quadrantes, verificamos que: 1) A ECS-UM e a FCS-UBI apresentam-se no quadrante inferior esquerdo, correspondente às Escolas com menor RET e menor número de ingressos; 2) O ICBAS surge no quadrante superior esquerdo, revelando baixo número de ingressos mas RET elevado; 3 ) A NMS|FCM, a FMUP e a FMUL (na transição) ocupam o quadrante inferior direito, apresentando elevado número de ingressos mas RET abaixo da média; 4) A FMUC aparece no quadrante superior direito, com elevado número de ingressos e RET acima da média.

\section{DISCUSSÃO}

O presente Estudo procurou caracterizar as condições pedagógicas das Escolas Médicas portuguesas, abrangendo quatro componentes de análise (ensino não clínico e clínico, condições de avaliação e de estudo), ao que acresce a identificação dos RET nas UCs clínicas. Esta caracterização assume particular relevância no contexto nacional de aumento do número de estudantes de Medicina ao longo dos últimos vinte anos. De facto, o incremento do número de estudantes admitidos nas Escolas Médicas em Portugal corresponde a $397 \%$ desde 1995, que coloca Portugal em primeiro lugar dos países da OCDE em aumento percentual no número de estudantes formados.

No ano letivo de 2013/2014, as Escolas Médicas no seu conjunto admitiram 1885 estudantes de Medicina, contabilizando todos os regimes de acesso. A FMUL, a FMUC, a FMUP e a NMS|FCM apresentam um número de ingressos superior à média nacional (226 estudantes), enquanto que o ICBAS, a ECS-UM, a FCS-UBI e o DCBM-UAlg estão abaixo da média nacional.

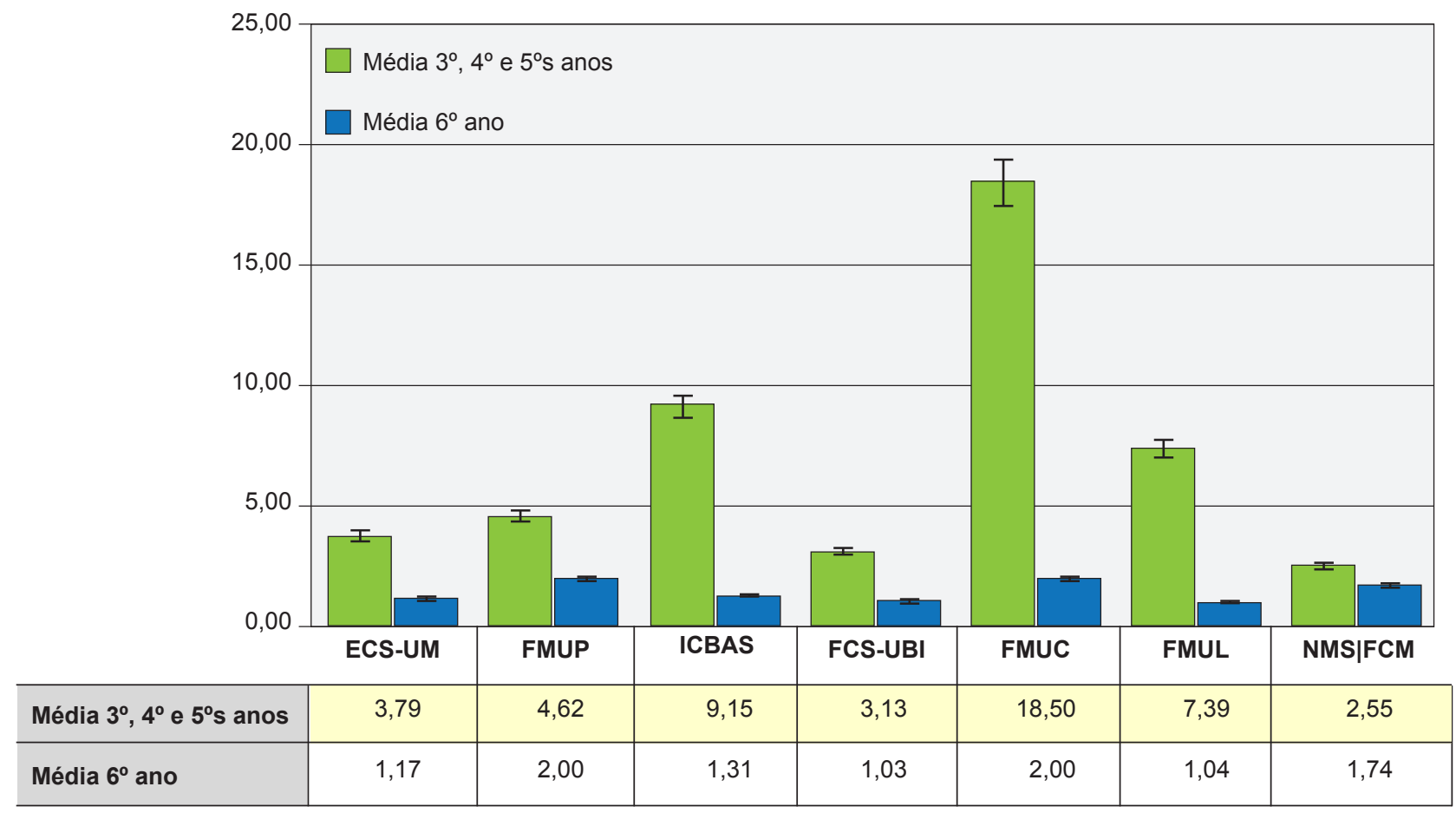

Figura 4 - Valores médios dos RET para os $3^{\circ}, 4^{\circ}$ e $5^{\circ} \mathrm{S}$ anos curriculares (a verde) e para o $6^{\circ}$ ano curricular (a azul), por Escola Médica. Intervalos de confiança a $95 \%$. 


\section{Satisfação Estudantil}

Os questionários de satisfação implementados nos anos de 2012 e 2013 permitem-nos caracterizar as condições pedagógicas das Escolas Médicas na perspetiva dos estudantes graças a uma amostra considerável de todos os anos curriculares, para um total de 3958 questionários nos dois anos de implementação. Já os RET permitem-nos analisar a componente sensível do ensino em meio clínico e assim tirar ilações sobre um dos espaços de maior valor na aquisição de competências no ensino da Medicina.

As condições de avaliação são o componente que mais satisfaz os estudantes, enquanto que o ensino em meio clínico é o componente de maior insatisfação em todas as Escolas Médicas. Globalmente, é possível verificar a existência de três realidades distintas na Educação Médica em Portugal: as Escolas com menor número de ingressos: ECS-UM, FCS-UBI e DCBM-UAlg apresentam maioritariamente os melhores resultados nos quatro componentes, enquanto que as Escolas com maior número de ingressos, nomeadamente a FMUP, FMUC, ICBAS, FMUC, FMUL e a NMS|FCM apresentam resultados inferiores. A FMUC destaca-se por apresentar tendencialmente os mais baixos índices de satisfação quanto às condições pedagógicas. Importa referir que o DCBM-UAlg apresenta uma metodologia de ensino distinta das restantes Escolas Médicas, adequada a estudantes já licenciados, pelo que a satisfação estudantil deve ser encarada à luz de uma Escola com estas características.

As diferenças entre Escolas Médicas traduzem o efeito de múltiplas variáveis. Entre estas, o número de ingressos anual é um determinante de relevo na Satisfação Global dos estudantes. Verifica-se que as Escolas com maior número de ingressos apresentam menor satisfação dos seus estudantes com o ensino médico. Existem no entanto Escolas em que, apesar de um número de ingressos superior (NMS|FCM e FMUP) ou inferior (ICBAS) à média nacional, apresentam, respetivamente, satisfação estudantil superior e inferior ao expectável pelo seu número de ingressos, o que traduz o efeito de variáveis não medidas. Estes resultados sugerem que algumas Escolas conseguem adequar, em maior ou menor grau, as suas condições pedagógicas ao número de ingressos anual, possivelmente através da otimização da distribuição do tempo letivo, da estruturação do ensino em meio clínico ou da qualidade e quantidade do corpo docente. Este fenómeno, que sugere que as Escolas têm mecanismos de adaptabilidade perante um elevado número de ingressos, verifica-se igualmente para os RET, em que algumas Escolas apresentam rácios superiores (ICBAS) ou inferiores (NMS|FCM e FMUP) ao esperado para o seu número de ingressos. Neste caso, os referidos mecanismos estão igualmente na dependência da articulação entre as Escolas e seus Hospitais de ensino afiliados.

As diferenças encontradas entre Escolas dependem também do efeito de variáveis específicas por cada componente de análise. No componente C2 (ensino em meio clínico) onde se obtêm os mais baixos resultados de satisfação

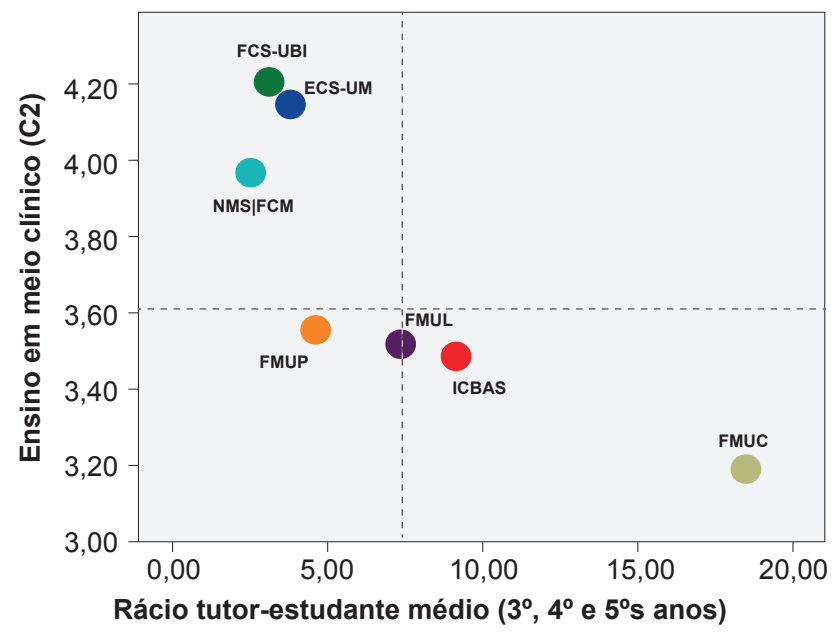

Figura 5 - Interação entre a pontuação média do componente C2 e os valores médios dos RET para o $3^{\circ}, 4^{\circ}$ e $5^{\circ} \mathrm{S}$ anos curriculares. A reta tracejada na vertical cruza o eixo das abcissas no valor médio dos RET das Escolas Médicas em Portugal (ponderado por número de ingressos) e a reta tracejada na horizontal cruza o eixo das ordenadas no valor médio do componente C2 nas Escolas Médicas (ponderado por número de ingressos).

estudantil, interagem variáveis tais como a interação estudante-tutor - que depende da qualidade do acompanhamento providenciado pelos médicos-docentes e dos RET (ver abaixo) - e a qualidade das experiências de ensino em meio clínico - que por sua vez dependem da capacidade formativa dos serviços hospitalares, incluindo enfermarias, consultas externas, serviço de urgência e bloco operatório.

De facto, o descontentamento com o ensino em meio clínico evidencia uma particular fragilidade na capacidade formativa das Escolas Médicas e Hospitais afiliados no que diz respeito ao ensino em meio clínico. Um elevado número estudantes a frequentar os cursos de Medicina tem um efeito expectavelmente superior no ensino em meio clínico do que no ensino não-clínico, já que coloca desafios à organização do tempo letivo e a um ensino personalizado em meio hospitalar. Isto coloca um maior desafio às Escolas criadas originalmente para acolher um número inferior de estudantes, por oposição às Escolas de criação mais recente. Esta contextualização permite interpretar a menor satisfação global dos estudantes nas Escolas Médicas com maior número de ingressos.

De facto, analisando todos os componentes, as condições pedagógicas agravam-se em conformidade com a progressão nos anos curriculares, o que pode dever-se a um maior descontentamento estudantil com o aumento proporcional de atividades letivas em meio clínico e maior consciencialização sobre as condições pedagógicas das Escolas e exigência subsequente com o ensino prestado e infraestruturas de apoio. No entanto, entre as Escolas Médicas, as mais recentes (DCBM-UAlg, FCS-UBI e ECSUM) não evidenciam um decréscimo da perceção sobre as condições pedagógicas ao longo dos anos curriculares, possivelmente devido a um ambiente educativo cujas condições pedagógicas são mais estáveis e mais ajustadas ao número de estudantes admitidos. 


\section{Rácios estudante-tutor}

No presente Estudo recolheram-se também os RET para as UCs com ensino em meio clínico. A literatura predominante e metodologias de análise das condições pedagógicas no ensino superior apresentam RET que associam ensino teórico e clínico, independentemente da estratégia de ensino-aprendizagem mobilizada. Estes RET têm valor num conjunto de indicadores sobre as condições pedagógicas das Escolas mas não nos dão um retrato das condições pedagógicas que atente à especificidade do ensino em meio clínico. Assim, procurou-se seguir uma abordagem distinta, calculando os RET de forma a representar um indicador mais fiável das condições em que decorre o ensino em meio clínico: analisou-se assim cada turma dentro de cada UC em cada ano curricular das várias Escolas Médicas, apenas entrando em conta com o sistema tutorial. Importa assinalar que o objetivo deste estudo não é inferir sobre a qualidade do complexo ensino em meio clinico através de uma única variável (os RET). Pretende-se obter uma estimativa dos RET que seja fiel à realidade por forma a inferir sobre as condições pedagógicas do ensino em meio clínico através de um indicador fiável. Para a análise das condições pedagógicas, a análise dos valores estimados de RET tem maior interesse por permitir realizar comparações entre Escolas e UCs, permitindo inferir quais as que apresentam diferenças estatisticamente significativas, e, consequentemente, quais as situações que merecem maior atenção.

Constata-se que existem diferenças estatisticamente significativas entre Escolas Médicas e entre as UCs dentro das mesmas Escolas. Comparando as várias instituições, podemos verificar que a FMUC se encontra destacada das restantes, com o mais elevado rácio estudante-tutor médio nos $3^{\circ}, 4^{\circ}$ e $5^{\circ} \mathrm{s}$ anos curriculares $(18,50)$. Já o ICBAS apresenta o segundo rácio médio mais elevado $(9,15)$, seguido da FMUL (7,39), FMUP $(4,62)$, ECS-UM $(3,79)$, FCS-UBI $(3,13)$, e, por fim, a NMS|FCM com os mais baixos RET $(2,55)$. Nos sextos anos curriculares, os RET variam entre 1,03 (FCS-UBI) e 2,00 (FMUP e FMUC), sem diferenças significativas entre Escolas.

A análise dos RET requer a apreciação de um conjunto de informações relevantes que contribuem tanto para a sobrevalorização como para subvalorização dos rácios calculados. Veja-se que no sexto ano curricular, dependendo da Escola Médica e da UC em causa, os estudantes são por vezes integrados em equipas médicas (com o seu tutor e outros médicos internos de especialidade e internos de ano comum), o que pode sobrevalorizar rácios estudante-tutor em UCs específicas. No entanto, dois outros fatores conduzem à subvalorização dos RET: 1) por um lado, o cálculo dos RET apenas contabiliza os estudantes da UC em causa que partilham um mesmo tutor, enquanto que os Hospitais de ensino pré-graduado assumem igualmente o ensino pós-graduado, pelo que os RET não entram em consideração com estudantes de outros anos curriculares ou mesmo internos do ano comum ou internos de especialidade que por vezes se encontram no mesmo ambiente de ensino, alocados ao mesmo tutor; 2) por outro, o cálculo dos RET entra em consideração com os docentes contratados bem como com docentes voluntários, sem vínculo contratual às Escolas Médicas. Acresce que um elevado número de estudantes por tutor pode traduzir-se, na prática, na transformação de aulas com contacto com doentes em aulas teórico-práticas sem contacto clínico ou na redução do tempo alocado ao contacto com pacientes. Importa também referir que a afiliação com outros Hospitais e Centros de Saúde apresenta-se como um importante mecanismo que as Escolas mobilizam, com maior ou menor impacto dependendo da instituição, para combater elevados RET e proporcionar experiências de ensino variadas aos seus estudantes.

Analisando a relação entre as pontuações médias das Escolas Médicas no componente C2 (ensino em meio clínico) e os seus RET médios ( $3^{\circ}, 4^{\circ}$ e $5^{\circ}$ s anos), as Escolas com maiores rácios apresentam menor satisfação estudantil com o ensino em meio clínico. Assim, os RET são uma variável importante na apreciação dos estudantes sobre o ensino clínico. De facto, não existem Escolas com satisfação estudantil elevada que apresentem um RET acima da média nacional. Pelo contrário, um RET abaixo do valor médio nacional não conduz necessariamente a satisfação com o ensino em meio clínico (veja-se o caso da FMUP - Fig. 5): sugerindo uma vez mais a relevância de outras variáveis como a qualidade do acompanhamento providenciado pelos tutores.

\section{CONCLUSÃO}

A satisfação estudantil e os rácios estudante-tutor têm valor enquanto indicadores de qualidade do ensino médico no contexto nacional. Neste estudo pioneiro em Portugal, foi possível identificar diferenças significativas entre diferentes componentes das condições pedagógicas: entre o ensino em meio não clínico, o ensino em meio clínico e as condições de estudo e avaliação, o ensino em meio clínico apresenta particular descontentamento por parte dos estudantes. Por outro lado, identificam-se disparidades entre a satisfação estudantil e rácios estudante-tutor entre Escolas Médicas e unidades curriculares: as Escolas com maior número de ingressos apresentam menor satisfação estudantil. No entanto, as instituições mostram diferentes capacidades de resposta e adaptabilidade tendo em conta o seu número de ingressos. O rácio estudante-tutor nacional é de 7,53, com diferenças estatisticamente significativas entre as várias Escolas (desde 18,5 na FMUC até 2,55 na NMS|FCM). Um rácio estudante-tutor elevado está diretamente correlacionado com menor satisfação estudantil com o ensino em meio clínico. A reduzida satisfação estudantil com o ensino em meio clínico associada a elevados rácios estudante-tutor em algumas Escolas Médicas sugerem compromisso da qualidade do ensino em meio clínico. Devem ser conduzidos esforços acrescidos para analisar de forma aprofundada a adequação das condições pedagógicas das Escolas, as disparidades significativas entre instituições e a qualidade do ensino médico em Portugal. 


\section{AGRADECIMENTOS}

Aos elementos do Grupo de Trabalho em Educação Médica de 2011, 2012 e 2013, aos estudantes de Medicina colaboradores, às Associações e Núcleos locais de estudantes de Medicina e às Escolas Médicas que prestaram apoio. A Patrício Costa e Milton Severo pela imprescindível colaboração.

\section{PROTECÇÃO DE PESSOAS E ANIMAIS}

Os autores declaram que os procedimentos seguidos estavam de acordo com os regulamentos estabelecidos pelos responsáveis da Comissão de Investigação Clínica e Ética e de acordo com a Declaração de Helsínquia da Associação Médica Mundial.

\section{REFERÊNCIAS}

1. Crisp N, Gawanas B, Sharp I; Task force for scaling up education and training for health workers. Training the health workforce: scaling up, saving lives. Lancet. 2008;371:689-91.

2. World Health Organization. Transforming and scaling up health professionals' education and training: World Health Organization Guidelines 2013. Geneva: WHO; 2013.

3. Review of Medical and Dental School Intakes in England. The health and education national strategic exchange (HENSE). Leeds: HENSE; 2012.

4. Basic Medical Education WFME Global Standards for Quality

\section{CONFIDENCIALIDADE DOS DADOS}

Os autores declaram ter seguido os protocolos do seu centro de trabalho acerca da publicação de dados.

\section{CONFLITOS DE INTERESSE}

Os autores declaram que não possuem conflitos de interesses.

\section{FONTES DE FINANCIAMENTO}

Os autores declaram não ter recebido subsídios ou bolsas para a elaboração do artigo.

Improvement, the 2012 revision. Copenhagen: World Federation for Medical Education; 2012.

5. Richardson JTE. Instruments for obtaining student feedback: a review of the literature. Assess Eval Higher Educ. 2005;30:387-415.

6. Higher Education Statistics Agency. Technical definitions: student:staff ratios (SSR) [consultado 2014 Nov 12]. Disponível em: https://www. hesa.ac.uk/component/content/article?id=2937.

7. Health at a Glance 2013: OECD Indicators. Organisation for Economic Cooperation and Development. Paris: OECD Publishing; 2013. 